PROCEEDINGS OF THE

AMERICAN MATHEMATICAL SOCIETY

Volume 134, Number 6, Pages 1759-1770

S 0002-9939(05)08199-2

Article electronically published on December 15, 2005

\title{
ON THE CONSTRUCTION OF FRAMES FOR TRIEBEL-LIZORKIN AND BESOV SPACES
}

\author{
GEORGE KYRIAZIS AND PENCHO PETRUSHEV
}

(Communicated by Andreas Seeger)

\begin{abstract}
We present a general method for construction of frames $\left\{\psi_{I}\right\}_{I \in \mathcal{D}}$ for Triebel-Lizorkin and Besov spaces, whose nature can be prescribed. In particular, our method allows for constructing frames consisting of rational functions or more general functions which are linear combinations of a fixed (small) number of shifts and dilates of a single smooth and rapidly decaying function $\theta$ such as the Gaussian $\theta(x)=\exp \left(-|x|^{2}\right)$. We also study the boundedness and invertibility of the frame operator $S f=\sum_{I \in \mathcal{D}}\left\langle f, \psi_{I}\right\rangle \psi_{I}$ on Triebel-Lizorkin and Besov spaces and give necessary and sufficient conditions for the dual system $\left\{S^{-1} \psi\right\}_{I \in \mathcal{D}}$ to be a frame as well.
\end{abstract}

\section{INTRODUCTION}

Frames were introduced in the early 1950's by Duffin and Schaeffer [DS] as a means for studying the convergence and summability properties of nonharmonic Fourier series. Since then frames have attracted a lot of attention, which is partly due to their numerous applications in Signal analysis, Fourier analysis, and Approximation theory. In particular, Gabor frames have been used for time-frequency analysis of functions (signals) (see [FG2]), while wavelet-type frames have been used in statistics, image processing, and nonlinear approximation (see e.g. [De, Do]).

Let $(H,\langle\cdot, \cdot\rangle)$ be a separable Hilbert space. A countable family of functions $\Psi \subset H$ is called a frame for $H$ if there exist constants $A, B>0$ such that for all $f \in H$,

$$
A\|f\|_{H}^{2} \leq \sum_{\psi \in \Psi}|\langle f, \psi\rangle|^{2} \leq B\|f\|_{H}^{2} .
$$

It is not hard to prove that the frame operator $S: H \rightarrow H$ defined by

$$
S f=\sum_{\psi \in \Psi}\langle f, \psi\rangle \psi
$$

is a bounded linear operator and $A I \leq S \leq B I$; hence $S$ is self-adjoint. Consequently, $S$ is invertible and $B^{-1} I \leq S^{-1} \leq A^{-1} I$. Moreover, the family $S^{-1} \Psi:=$ $\left\{S^{-1} \psi\right\}_{\psi \in \Psi}$ is a frame for $H$ as well. Further, for every $f \in H$,

$$
f=S S^{-1} f=\sum_{\psi \in \Psi}\left\langle f, S^{-1} \psi\right\rangle \psi .
$$

Received by the editors July 6, 2004 and, in revised form, January 24, 2005.

2000 Mathematics Subject Classification. Primary 42C15, 46E99, 46B15, 41A63, 94A12.

The second author was supported by the National Science Foundation Grant DMS-0200665. 
Thus $\Psi$ provides a stable representation of all $f \in H$; it acts like a Riesz basis. However, unlike bases, in general, $\Psi$ is redundant. Thus representation (1.1) is not necessarily unique. In many applications this redundancy turns out to be of an advantage since it provides extra flexibility. We refer the reader to [HW] for a more detailed overview of the basic properties of frames for Hilbert spaces.

In the late 1980's it was recognized that certain elements of the abstract theory on Hilbert spaces could be extended to more general settings. Gröchenig [G], building upon the theory of the so-called co-orbit spaces and using group theoretic techniques, introduced frames for Banach spaces which included the modulation spaces and the Besov-Triebel-Lizorkin spaces (see also [FG1]).

Suppose that $X$ is a quasi-Banach space and let $Y$ be an associated sequence space. We say that a countable family of functions $\Psi$ in the dual $X^{*}$ of $X$ is a frame for $X$ if there exist constants $A, B>0$ such that for all $f \in X$,

$$
A\|f\|_{X} \leq\left\|(\langle f, \psi\rangle)_{\psi \in \Psi}\right\|_{Y} \leq B\|f\|_{X},
$$

where $\langle f, \psi\rangle:=\psi(f)$.

Our goal in this article is to develop a general method for construction of frames for homogeneous Triebel-Lizorkin and Besov spaces. An obvious modification of this method (which will not be considered here) produces frames for inhomogeneous Triebel-Lizorkin and Besov spaces as well. Our method utilizes a specific small perturbation principle which allows for a lot of flexibility. Thus the main feature of our method is that the nature of the frame elements can be prescribed. In particular, if $\theta$ is a sufficiently smooth and rapidly decaying function on $\mathbb{R}^{d}$, then our method allows for constructing a frame consisting of functions which are linear combinations of a fixed (small) number of shifts and dilates of $\theta$. Typical examples of such functions are $\theta(x)=\exp \left(-|x|^{2}\right)$ and $\theta(x)=\left(1+|x|^{2}\right)^{-N}$. For construction of bases, this method was first introduced in Pet and further generalized and refined in $\underline{\mathrm{KP}}$.

Frames for Triebel-Lizorkin-Besov spaces can be valuable if the representation (1.1) holds; this is no longer a byproduct of the general theory. Moreover, it is important to know whether the membership of a distribution $f$ to a certain Triebel-Lizorkin or Besov space can be characterized by the frame coefficients $\left\langle f, S^{-1} \psi\right\rangle, \psi \in \Psi$, i.e. if $S^{-1} \Psi$ is a frame itself. These are the primary objectives of our development of new frames in this article.

To better describe our results we begin with the introduction of some standard notation. As is customary we denote by $\mathbb{S}:=\mathbb{S}\left(\mathbb{R}^{d}\right)$ the Schwartz space of infinitely differentiable, rapidly decreasing functions on $\mathbb{R}^{d}$ and by $\mathbb{S}^{\prime}:=\mathbb{S}^{\prime}\left(\mathbb{R}^{d}\right)$ its dual, the space of tempered distributions. We also denote by $\mathbb{S}^{\prime} / \mathcal{P}$ the space of equivalence classes of distributions in $\mathbb{S}^{\prime}$ modulo polynomials, and by $\mathbb{S}_{\infty}$ its dual (for more details and references we refer the reader to $[\mathrm{Gr}],[\mathrm{K}], \mathrm{T}]$ ).

We let $\mathcal{D}$ denote the family of all dyadic cubes in $\mathbb{R}^{d}$ and let $\mathcal{D}_{m}, m \in \mathbb{Z}$, be the collection of all cubes $I \in \mathcal{D}$ of side-length $\ell(I)=2^{-m}$. For any dyadic cube $I \in \mathcal{D}$, we use $x_{I}$ to denote its lower-left corner and $|I|$ for its volume.

As usual the Fourier transform $\widehat{f}$ of an integrable function $f$ is defined by $\widehat{f}(\xi)=$ $\int_{\mathbb{R}^{d}} f(x) e^{-i x \xi} d x$ and can be extended by duality uniquely from $\mathbb{S}$ to $\mathbb{S}^{\prime}$. Finally, we use $\langle f, \eta\rangle$ for the standard inner product $\int f \bar{\eta}$ of two functions (whenever this makes sense) and the same notation is employed for the action of a distribution $f \in \mathbb{S}^{\prime}$ on $\bar{\eta} \in \mathbb{S}$. 
1.1. Frames for homogeneous Triebel Lizorkin and Besov spaces. Choose an arbitrary function $\varphi \in \mathbb{S}$ so that $\operatorname{supp} \widehat{\varphi} \subset\left\{\xi: 2^{-1} \leq|\xi| \leq 2\right\}$ and $|\widehat{\varphi}(\xi)| \geq c>0$ if $3 / 5 \leq|\xi| \leq 5 / 3$, and define $\varphi_{\nu}(\cdot):=2^{d \nu} \varphi\left(2^{\nu} \cdot\right)$.

For $s \in \mathbb{R}, 0<p<\infty, 0<q \leq \infty$, the homogeneous Triebel-Lizorkin space $\dot{F}_{p q}^{s}$ is defined as the set of all $f \in \mathbb{S}^{\prime} / \mathcal{P}$ such that

$$
\|f\|_{\dot{F}_{p q}^{s}}:=\left\|\left(\sum_{\nu \in \mathbb{Z}}\left[2^{\nu s}\left|\varphi_{\nu} * f\right|\right]^{q}\right)^{1 / q}\right\|_{L_{p}}<\infty
$$

with the usual modification when $q=\infty$. By interchanging the roles of integration and summation we get the homogeneous Besov spaces; that is, for $s \in \mathbb{R}, 0<p, q \leq$ $\infty, \dot{B}_{p q}^{s}$ is defined as the set of all $f \in \mathbb{S}^{\prime} / \mathcal{P}$ such that

$$
\|f\|_{\dot{B}_{p q}^{s}}:=\left(\sum_{\nu \in \mathbb{Z}}\left[2^{\nu s}\left\|\varphi_{\nu} * f\right\|_{L_{p}}\right]^{q}\right)^{1 / q}<\infty .
$$

It can be shown that the above definitions of Triebel-Lizorkin and Besov spaces are independent of the specific selection of $\varphi$ (see e.g. [T]).

Note that the F- and B-spaces are quasi-Banach spaces (Banach spaces if $p, q \geq$ 1 ) and by varying the indexes $s, p, q$ one can recover from them most of the classical spaces (see e.g. [T]). In particular, $\dot{F}_{p 2}^{0} \approx L_{p}$ if $1<p<\infty$, and $\dot{F}_{p 2}^{0} \approx H_{p}$ (the Hardy space) if $0<p \leq 1$. Also, for $s>0,1<p<\infty$, $\dot{F}_{p 2}^{s}$ coincides with the potential space $H_{p}^{s}$ and for integer values of $s, \dot{F}_{p 2}^{s} \approx W_{p}^{s}$, the Sobolev space equipped with its seminorm. Here $\approx$ means that the spaces have equivalent (quasinorms.

Now, let $\varphi \in \mathbb{S}$ satisfy

(i) $\operatorname{supp} \widehat{\varphi} \subset\left\{\xi: 2^{-1} \leq|\xi| \leq 2\right\}$,

(ii) $|\widehat{\varphi}(\xi)| \geq c>0$ if $3 / 5 \leq|\xi| \leq 5 / 3$,

$$
\text { (iii) } \sum_{\nu \in \mathbb{Z}}\left|\widehat{\varphi}\left(2^{\nu} \xi\right)\right|^{2}=1, \quad \xi \neq 0 \text {. }
$$

By $\left[1.2\left(\right.\right.$ iii) ) it readily follows that for $f \in \mathbb{S}^{\prime} / \mathcal{P}$,

$$
\sum_{\nu \in \mathbb{Z}} \widetilde{\varphi}_{\nu} * \varphi_{\nu} * f=f, \quad \widetilde{\varphi}_{\nu}(x):=\overline{\varphi_{\nu}(-x)},
$$

in the sense of $\mathbb{S}^{\prime} / \mathcal{P}$. Identity (1.3) is the so-called Calderón reproducing formula. Using techniques reminiscent of the Shannon sampling theorem and expanding $\widetilde{\varphi}_{\nu} * \varphi_{\nu} * f$, one can further show [FJ2] that for all $f \in \mathbb{S}^{\prime} / \mathcal{P}$,

$$
f=\sum_{I \in \mathcal{D}}\left\langle f, \varphi_{I}\right\rangle \varphi_{I}
$$

where $\varphi_{I}(\cdot):=|I|^{-1 / 2} \varphi\left(\frac{-x_{I}}{\ell(I)}\right)$.

The F- and B-spaces are naturally characterized by the sequence spaces $\dot{f}_{p q}^{s}$ and $\dot{b}_{p q}^{s}$ defined as follows: For $s \in \mathbb{R}, 0<p<\infty$, and $0<q \leq \infty$,

$$
\dot{f}_{p q}^{s}:=\left\{h:=\left(h_{I}\right)_{I \in \mathcal{D}}:\|h\|_{\dot{f}_{p q}^{s}}<\infty\right\},
$$

where

$$
\|h\|_{\dot{f}_{p q}^{s}}:=\left\|\left(\sum_{I \in \mathcal{D}}\left(|I|^{-s / d-1 / 2}\left|h_{I} \mathbb{1}_{I}\right|\right)^{q}\right)^{1 / q}\right\|_{L_{p}} .
$$


Similarly, for $s \in \mathbb{R}$ and $0<p, q \leq \infty$,

$$
\dot{b}_{p q}^{s}:=\left\{h:=\left(h_{I}\right)_{I \in \mathcal{D}}:\|h\|_{\dot{b}_{p q}^{s}}<\infty\right\},
$$

where

$$
\|h\|_{\dot{b}_{p q}^{s}}:=\left(\sum_{e \in E} \sum_{m \in \mathbb{Z}}\left(\sum_{I \in \mathcal{D}_{m}}\left(|I|^{-s / d+1 / p-1 / 2}\left|h_{I e}\right|\right)^{p}\right)^{q / p}\right)^{1 / q} .
$$

A fundamental result due to Frazier and Jawerth [FJ1, FJ2] states that the family $\Phi:=\left\{\varphi_{I}\right\}_{I \in \mathcal{D}}$ is a frame for both the Triebel-Lizorkin and Besov spaces: If $s \in \mathbb{R}, 0<p<\infty$, and $0<q \leq \infty$, there exist constants $c_{1}, c_{2}>0$ such that for $f \in \dot{F}_{p q}^{s}$,

$$
c_{1}\|f\|_{\dot{F}_{p q}^{s}} \leq\left\|\left(\left\langle f, \varphi_{I}\right\rangle\right)_{I}\right\|_{\dot{f}_{p q}^{s}} \leq c_{2}\|f\|_{\dot{F}_{p q}^{s}} .
$$

Similarly, if $s \in \mathbb{R}$, and $0<p, q \leq \infty$, then for $f \in \dot{B}_{p q}^{s}$,

$$
c_{1}\|f\|_{\dot{B}_{p q}^{s}} \leq\left\|\left(\left\langle f, \varphi_{I}\right\rangle\right)_{I}\right\|_{\dot{b}_{p q}^{s}} \leq c_{2}\|f\|_{\dot{B}_{p q}^{s}} .
$$

1.2. The construction of new frames. The construction of affine frames in the literature follows in the footsteps of the main scheme for constructing wavelet bases, namely, by making use of the notion of a Multiresolution analysis (see e.g. [BL]). As a consequence, most of the constructed frames are of a very particular nature.

Here instead we propose a method for constructing frames based on the idea of the small perturbation principle. The starting point of our method is the selection of a frame $\Phi:=\left\{\varphi_{I}\right\}_{I \in \mathcal{D}}$ such as the Frazier-Jawerth frame described above, consisting of sufficiently smooth and rapidly decaying functions. Then, the main step of our scheme is to approximate every frame element $\varphi_{I}$ of $\Phi$ by a function $\psi_{I}$ of the new system $\Psi:=\left\{\psi_{I}\right\}_{I \in \mathcal{D}}$.

The grand question is: What kind of approximation should be used in this construction in order that the new system $\Psi$ be a frame for the spaces of interest?

Following the idea introduced in Pet and further developed in $\mathrm{KP}$, we propose the following approximation scheme: For suitable values of the parameters $\varepsilon, r, k, M>0$ we require that all $\psi_{I}$ in $\Psi$ satisfy the conditions:

A1.

$$
\left|\partial^{\alpha} \varphi_{I}(x)-\partial^{\alpha} \psi_{I}(x)\right| \leq \varepsilon|I|^{-1 / 2-|\alpha| / d}\left(1+\ell(I)^{-1}\left|x-x_{I}\right|\right)^{-M}, \quad|\alpha| \leq r .
$$

A2.

$$
\int_{\mathbb{R}^{d}} x^{\alpha} \psi_{I}(x) d x=0, \quad|\alpha| \leq k .
$$

Here the $\psi_{I}$ 's are not necessarily the dyadic dilates and shifts of a single function. For simplicity we shall assume in the following that $\Phi$ is the Frazier-Jawerth frame described above. However, any other frame consisting of sufficiently smooth and rapidly decaying functions with enough vanishing moments can do the job.

We shall prove in Theorem 3.1 that if $\varepsilon>0$ is sufficiently small and $r, k, M$ are sufficiently large (depending on $s, p, q$ ), then the family $\Psi$ is a frame for the BesovTriebel-Lizorkin spaces. The questions that naturally arise here are, whether $S^{-1} \Psi$ is a frame itself and if the representation

$$
f=\sum_{I \in \mathcal{D}}\left\langle f, S^{-1} \psi_{I}\right\rangle \psi_{I}
$$


holds true? It turns out that the key to both questions is the boundedness of the operator with matrix

$$
\mathbf{B}=\left(b_{I J}\right)_{I, J \in \mathcal{D}}, \quad \text { where } b_{I J}:=\left\langle\varphi_{J}, S^{-1} \psi_{I}\right\rangle,
$$

on $\dot{f}_{p q}^{s}$ (or $\dot{b}_{p q}^{s}$ ). As we show in Theorem 3.3 the boundedness of $\mathbf{B}$ is a necessary and sufficient condition for $S^{-1} \Psi$ to be a frame for the Triebel-Lizorkin and Besov spaces. Moreover, the same condition guarantees that (1.7) holds in the distributional sense.

Since the family $\Phi$ is generated by dilations and shifts of a single function $\varphi$, it suffices to approximate $\varphi$ by a function $\psi$ satisfying the conditions:

B1.

$$
\left|\partial^{\alpha} \varphi(x)-\partial^{\alpha} \psi(x)\right| \leq \varepsilon(1+|x|)^{-M}, \quad|\alpha| \leq r .
$$

B2.

$$
\int_{\mathbb{R}^{d}} x^{\alpha} \psi(x) d x=0, \quad|\alpha| \leq k .
$$

Then we define the new system $\Psi$ by $\Psi:=\left\{\psi_{I}\right\}_{I \in \mathcal{D}}$, where $\psi_{I}(\cdot):=|I|^{-1 / 2} \psi\left(\frac{\cdot x_{I}}{\ell(I)}\right)$.

The construction of functions $\psi$ satisfying $\mathbf{B 1}$ and $\mathbf{B 2}$ has been explored in $\mathrm{KP}$. In particular, it is shown that if $\theta$ is an arbitrary sufficiently smooth and rapidly decaying function on $\mathbb{R}^{d}$, then one can devise $\psi$ satisfying $\mathbf{B 1}$ and $\mathbf{B 2}$ using a finite linear combination of shifts and dilates of $\theta$. Typical examples include the rational function $\theta(\cdot):=\left(1+|\cdot|^{2}\right)^{-N}$ and the Gaussian $\theta(\cdot):=e^{-|\cdot|^{2}}$.

\section{Auxiliary Results}

In this section we present some basic results concerning the algebra of almost diagonal operators on $\dot{f}_{p q}^{s}$ and $\dot{b}_{p q}^{s}$ and their interplay with the study of the boundedness of linear operators on Triebel-Lizorkin and Besov spaces. These results have been established by Frazier and Jawerth within the framework of the so-called $\varphi$ transform (see [FJ1, FJ2, FJW]) and will be needed for our developments in the next section.

Let $\mathbf{A}$ be a linear operator acting on $\dot{f}_{p q}^{s}$ or $\dot{b}_{p q}^{s}$ with associated matrix $\left(a_{I J}\right)_{I, J \in \mathcal{D}}$. We say that $\mathbf{A}$ is almost diagonal on $\dot{f}_{p q}^{s}$ or $\dot{b}_{p q}^{s}$ if there exist $\delta>0$ and $c>0$ such that

$$
\left|a_{I J}\right| \leq c \omega_{\delta}(I, J), \quad I, J \in \mathcal{D},
$$

with

$$
\begin{aligned}
\omega_{\delta}(I, J) & :=\left(\frac{\ell(I)}{\ell(J)}\right)^{s}\left(1+\frac{\left|x_{I}-x_{J}\right|}{\max \{\ell(I), \ell(J)\}}\right)^{-\mathcal{J}-\delta} \\
& \times \min \left\{\left(\frac{\ell(I)}{\ell(J)}\right)^{(d+\delta) / 2},\left(\frac{\ell(J)}{\ell(I)}\right)^{(\delta-d) / 2+\mathcal{J}}\right\},
\end{aligned}
$$

where $\mathcal{J}:=d / \min \{1, p, q\}$ for $\dot{f}_{p q}^{s}$ and $\mathcal{J}:=d / \min \{1, p\}$ for $\dot{b}_{p q}^{s}$.

We denote by $\mathbf{a d}_{p q}^{s}$ the collection of all almost diagonal operators on $\dot{f}_{p q}^{s}$ or $\dot{b}_{p q}^{s}$. It turns out that $\mathbf{a d}_{p q}^{s}$ is closed under composition and therefore it is an algebra. Moreover, almost diagonal operators are bounded on $\dot{f}_{p q}^{s}$ and $\dot{b}_{p q}^{s}$.

Proposition 2.1. Let $s \in \mathbb{R}, 0<p, q \leq \infty$ and $\mathbf{A} \in \mathbf{a d}_{p q}^{s}$. Then $\mathbf{A}$ is bounded on $\dot{b}_{p q}^{s}$ and, if $p<\infty$, on $\dot{f}_{p q}^{s}$. 
It is not hard to construct almost diagonal operators for the $f$ - or $b$-spaces. Consider for instance the following construction: Let $\mathcal{J}$ be as above and $\mathcal{N}:=$ $\max \{[\mathcal{J}-d-s],-1\}$. Suppose that for some $M>\max \{\mathcal{J}, d+s\}, k \geq[s]$, and $r>\mathcal{N}$, the functions $\left\{b_{I}\right\}_{I \in \mathcal{D}}$ satisfy the conditions

$$
\begin{aligned}
& \left|\partial^{\alpha} b_{I}(x)\right| \leq c|I|^{-1 / 2-|\alpha| / d}\left(1+\ell(I)^{-1}\left|x-x_{I}\right|\right)^{-M}, \quad|\alpha| \leq r, \\
& \int_{\mathbb{R}^{d}} x^{\alpha} b_{I}(x) d x=0, \quad|\alpha| \leq k,
\end{aligned}
$$

where (2.2) is void if $s<0$, then the operator $\mathbf{A}$ with matrix

$$
\mathbf{A}=\left(a_{I J}\right)_{I, J \in \mathcal{D}}, \text { where } a_{I J}:=\left\langle\varphi_{J}, b_{I}\right\rangle,
$$

is almost diagonal on $\dot{f}_{p q}^{s}$ or $\dot{b}_{p q}^{s}$.

An important consequence of the boundedness of $\mathbf{A}$ is that the family $\left\{b_{I}\right\}_{I \in \mathcal{D}}$ is a norming family for the Triebel-Lizorkin and Besov spaces; that is, for all $f \in \dot{F}_{p q}^{s}$,

$$
\left\|\left(\left\langle f, b_{I}\right\rangle\right)_{I}\right\|_{\dot{f}_{p q}^{s}} \leq c\|f\|_{\dot{F}_{p q}^{s}},
$$

and similarly for $f \in \dot{B}_{p q}^{s}$,

$$
\left\|\left(\left\langle f, b_{I}\right\rangle\right)_{I}\right\|_{\dot{b}_{p q}^{s}} \leq c\|f\|_{\dot{B}_{p q}^{s}} .
$$

We say that $\left\{m_{I}\right\}_{I \in \mathcal{D}}$ is a family of smooth molecules for $\dot{F}_{p q}^{s}$ or $\dot{B}_{p q}^{s}$ if for some $r>[s], k \geq \mathcal{N}$, and $M>\mathcal{J}$,

$$
\begin{aligned}
& \left|m_{I}(x)\right| \leq c|I|^{-1 / 2}\left(1+\ell(I)^{-1}\left|x-x_{I}\right|\right)^{-\max (M, M-s)}, \\
& \left|\partial^{\alpha} m_{I}(x)\right| \leq c|I|^{-1 / 2-|\alpha| / d}\left(1+\ell(I)^{-1}\left|x-x_{I}\right|\right)^{-M}, \quad|\alpha| \leq r, \\
& \int_{\mathbb{R}^{d}} x^{\alpha} m_{I}(x) d x=0, \quad|\alpha| \leq k,
\end{aligned}
$$

where (2.7) is void if $s<0$.

Similarly as above, molecules give rise to almost diagonal operators. For instance, the operator $\mathbf{C}$ with matrix

$$
\mathbf{C}=\left(c_{I J}\right)_{I, J \in \mathcal{D}}, \text { where } c_{I J}:=\left\langle m_{J}, \varphi_{I}\right\rangle,
$$

is almost diagonal on $\dot{f}_{p q}^{s}$ and $\dot{b}_{p q}^{s}$. Consequently, for every sequence $\left(s_{I}\right)_{I} \in \dot{f}_{p q}^{s}$,

$$
\left\|\sum_{I} s_{I} m_{I}\right\|_{\dot{F}_{p q}^{s}} \leq c\left\|\left(s_{I}\right)_{I}\right\|_{\dot{f}_{p q}^{s}}
$$

and similarly if $\left(s_{I}\right)_{I} \in \dot{b}_{p q}^{s}$, then

$$
\left\|\sum_{I} s_{I} m_{I}\right\|_{\dot{B}_{p q}^{s}} \leq c\left\|\left(s_{I}\right)_{I}\right\|_{\dot{b}_{p q}^{s}} .
$$

Let us now assume that $T$ is a continuous linear operator from $\mathbb{S}$ to $\mathbb{S}^{\prime}$. If the operator with matrix

$$
\mathbf{D}=\left(d_{I J}\right)_{I, J \in \mathcal{D}}, \text { where } d_{I J}:=\left\langle T \varphi_{J}, \varphi_{I}\right\rangle,
$$


is almost diagonal on $\dot{f}_{p q}^{s}$ or $\dot{b}_{p q}^{s}$, then $T$ extends to a bounded operator on $\dot{F}_{p q}^{s}$ or $\dot{B}_{p q}^{s}$. Indeed, employing (1.4)-(1.5), we have for $f \in \dot{F}_{p q}^{s}$,

$$
\begin{aligned}
\|T f\|_{\dot{F}_{p q}^{s}} & \leq c\left\|\left(\left\langle T f, \varphi_{I}\right\rangle\right)_{I}\right\|_{\dot{f}_{p q}^{s}}=c\left\|\left(\sum_{J} d_{I J}\left\langle f, \varphi_{J}\right\rangle\right)_{I}\right\|_{\dot{f}_{p q}^{s}} \\
& \leq c\left\|\left(\left\langle f, \varphi_{I}\right\rangle\right)_{I}\right\|_{\dot{f}_{p q}^{s}} \leq c\|f\|_{\dot{F}_{p q}^{s}} .
\end{aligned}
$$

A similar result holds for Besov spaces as well.

\section{Main Results}

We recall some of our notation from the previous section: $\mathcal{J}:=d / \min \{1, p, q\}$ in the case of Triebel-Lizorkin spaces, while $\mathcal{J}:=d / \min \{1, p\}$ for Besov spaces. Also $\mathcal{N}:=\max \{[\mathcal{J}-d-s],-1\}$.

Theorem 3.1. Suppose that $s \in \mathbb{R}, 0<p, q \leq \infty$ and let $\Psi:=\left\{\psi_{I}\right\}_{I \in \mathcal{D}}$ satisfy $\mathbf{A} 1$ and $\mathbf{A 2}$ for some $r>\mathcal{N}, k \geq[s]$, and $M>\max \{\mathcal{J}, d+s\}$. If $\varepsilon>0$ is sufficiently small, then there exist constants $A, B>0$ such that for $f \in \dot{B}_{p q}^{s}$,

$$
A\|f\|_{\dot{B}_{p q}^{s}} \leq\left\|\left(\left\langle f, \psi_{I}\right\rangle\right)_{I}\right\|_{\dot{b}_{p q}^{s}} \leq B\|f\|_{\dot{B}_{p q}^{s}}
$$

and, if $p<\infty$, for $f \in \dot{F}_{p q}^{s}$,

$$
A\|f\|_{\dot{F}_{p q}^{s}} \leq\left\|\left(\left\langle f, \psi_{I}\right\rangle\right)_{I}\right\|_{\dot{f}_{p q}^{s}} \leq B\|f\|_{\dot{F}_{p q}^{s}} .
$$

Proof. We shall prove only (3.2) since (3.1) follows similarly. From A1 and A2 it follows that $\Psi$ is a norming family for $\dot{F}_{p q}^{s}$ (see (2.4) ), i.e. there exists a constant $B$ such that for $f \in \dot{F}_{p q}^{s}$

$$
\left\|\left(\left\langle f, \psi_{I}\right\rangle\right)_{I}\right\|_{\dot{f}_{p q}^{s}} \leq B\|f\|_{\dot{F}_{p q}^{s}} .
$$

Thus we only have to establish the left-hand-side estimate in (3.2). For this we note that since $\left(\varphi_{I}\right)$ satisfies (2.1)-(2.2) for all $r>0$, the family $\left(\left(\varphi_{I}-\psi_{I}\right) \varepsilon^{-1}\right)$ is a norming family as well and, therefore, for some $c>0$,

$$
\left\|\left(\left\langle f, \varphi_{I}-\psi_{I}\right\rangle\right)_{I}\right\|_{\dot{f}_{p q}^{s}} \leq c \varepsilon\|f\|_{\dot{F}_{p q}^{s}} .
$$

Consequently,

$$
\begin{aligned}
\left\|\left(\left\langle f, \varphi_{I}\right\rangle\right)_{I}\right\|_{\dot{f}_{p q}^{s}} & \leq c\left(\left\|\left(\left\langle f, \psi_{I}\right\rangle\right)_{I}\right\|_{\dot{f}_{p q}^{s}}+\left\|\left(\left\langle f, \varphi_{I}-\psi_{I}\right\rangle\right)_{I}\right\|_{\dot{f}_{p q}^{s}}\right) \\
& \leq c\left(\left\|\left(\left\langle f, \psi_{I}\right\rangle\right)_{I}\right\|_{\dot{f}_{p q}^{s}}+\varepsilon\|f\|_{\dot{F}_{p q}^{s}}\right) .
\end{aligned}
$$

Taking into account that the family $\Phi$ is a frame, using (1.5) we obtain

$$
c_{1}\|f\|_{\dot{F}_{p q}^{s}} \leq c\left\|\left(\left\langle f, \psi_{I}\right\rangle\right)_{I}\right\|_{\dot{f}_{p q}^{s}}+c \varepsilon\|f\|_{\dot{F}_{p q}^{s}} .
$$

Choosing $\varepsilon>0$ sufficiently small, we have $A:=\left(c_{1}-c \varepsilon\right) / c>0$ and hence

$$
A\|f\|_{\dot{F}_{p q}^{s}} \leq\left\|\left(\left\langle f, \psi_{I}\right\rangle\right)_{I}\right\|_{\dot{f}_{p q}^{s}},
$$

which completes the proof of the theorem.

Assume now that $\Psi:=\left\{\psi_{I}\right\}_{I \in \mathcal{D}}$ is a frame for $\dot{F}_{p q}^{s}$ (or $\dot{B}_{p q}^{s}$ ) satisfying (2.1)-(2.2) (with $\left\{b_{I}\right\}$ replaced by $\left\{\psi_{I}\right\}$ ) and let $k, M, r$ be sufficiently large. If $k \geq 0, M>d$, 
and $r>0, \Psi$ constitutes a frame for $F_{22}^{0}=B_{22}^{0}=L_{2}\left(\mathbb{R}^{d}\right)$ and from the Hilbert space theory we know that the frame operator $S: L_{2}\left(\mathbb{R}^{d}\right) \rightarrow L_{2}\left(\mathbb{R}^{d}\right)$ defined by

$$
S f:=\sum_{I \in \mathcal{D}}\left\langle f, \psi_{I}\right\rangle \psi_{I}
$$

is self-adjoint and invertible. Moreover, the family $S^{-1} \Psi:=\left\{S^{-1} \psi_{I}\right\}_{I \in \mathcal{D}}$ is also a frame for $L_{2}\left(\mathbb{R}^{d}\right)$ and for $f \in L_{2}\left(\mathbb{R}^{d}\right)$,

$$
f=\sum_{I \in \mathcal{D}}\left\langle f, S^{-1} \psi_{I}\right\rangle \psi_{I}
$$

We would like to devise conditions on $\Psi$ which would guarantee that $S^{-1} \Psi$ is a frame for the Triebel-Lizorkin and Besov spaces as well. As already mentioned in the introduction, in essence one has to study the boundedness of the operator $\mathbf{B}$ with matrix

$$
\mathbf{B}:=\left(b_{I J}\right)_{I, J \in \mathcal{D}}, \quad \text { where } b_{I J}:=\left\langle\varphi_{J}, S^{-1} \psi_{I}\right\rangle,
$$

on the corresponding sequence spaces.

We first have to give meaning to the coefficients $\left\langle f, S^{-1} \psi_{I}\right\rangle$. Since $S^{-1} \Psi$ is a frame for $L_{2}\left(\mathbb{R}^{d}\right)$, if $f \in L_{2}\left(\mathbb{R}^{d}\right)$, then $f=\sum_{J}\left\langle f, \varphi_{J}\right\rangle \varphi_{J}$ in $L_{2}$, and hence

$$
\left\langle f, S^{-1} \psi_{I}\right\rangle=\sum_{J \in \mathcal{D}}\left\langle f, \varphi_{J}\right\rangle\left\langle\varphi_{J}, S^{-1} \psi_{I}\right\rangle, \quad I \in \mathcal{D} .
$$

Assuming that B is bounded on $\dot{f}_{p q}^{s}$, (3.4) yields that for $f \in L_{2}\left(\mathbb{R}^{d}\right) \cap \dot{F}_{p q}^{s}$ and $I \in \mathcal{D}$,

$$
\left|\left\langle f, S^{-1} \psi_{I}\right\rangle\right| \leq\left\|\left(\left\langle f, S^{-1} \psi_{I}\right\rangle\right)_{I}\right\|_{\dot{f}_{p q}^{s}} \leq c\left\|\left(\left\langle f, \varphi_{I}\right\rangle\right)_{I}\right\|_{\dot{f}_{p q}^{s}} \leq c\|f\|_{\dot{F}_{p q}^{s}} .
$$

A similar argument applies to the $\dot{b}_{p q}^{s}$ and $\dot{B}_{p q}^{s}$ spaces as well. Using the density of $L_{2}\left(\mathbb{R}^{d}\right)$ in $\dot{F}_{p q}^{s}$ (or $\dot{B}_{p q}^{s}$ ) and a standard argument, one now easily extends the linear functional $S^{-1} \psi_{I}: f \rightarrow\left\langle f, S^{-1} \psi_{I}\right\rangle$ to $\dot{F}_{p q}^{s}\left(\right.$ or $\left.\dot{B}_{p q}^{s}\right)$.

Lemma 3.2. Suppose that $s \in \mathbb{R}, 0<p, q \leq \infty$ and let $\Psi:=\left\{\psi_{I}\right\}_{I \in \mathcal{D}}$ be a frame for $L_{2}\left(\mathbb{R}^{d}\right)$ satisfying (2.6)-(2.8) for some $r>[s], k \geq \mathcal{N}$, and $M>\mathcal{J}$. If $\mathbf{B}$ is bounded on $\dot{b}_{p q}^{s}$, then for $f \in \dot{B}_{p q}^{s}$,

$$
f=\sum_{I \in \mathcal{D}}\left\langle f, S^{-1} \psi_{I}\right\rangle \psi_{I}
$$

in the sense of $\mathbb{S}^{\prime} / \mathcal{P}$. Similarly, if $p<\infty$ and $\mathbf{B}$ is bounded on $\dot{f}_{p q}^{s}$, then (3.6) holds for $f \in \dot{F}_{p q}^{s}$.

Proof. We shall again treat only the case of Triebel-Lizorkin spaces. Since $\Psi$ is a frame for $L_{2}\left(\mathbb{R}^{d}\right)$, we know that for every $I \in \mathcal{D}$,

$$
\varphi_{I}=\sum_{J \in \mathcal{D}}\left\langle\varphi_{I}, S^{-1} \psi_{J}\right\rangle \psi_{J}
$$

in $L_{2}$ (and consequently in the sense of $\mathbb{S}^{\prime} / \mathcal{P}$ ). 
For $f \in \dot{F}_{p q}^{s}$, we have

$$
\begin{aligned}
f & =\sum_{I \in \mathcal{D}}\left\langle f, \varphi_{I}\right\rangle \varphi_{I}=\sum_{I \in \mathcal{D}} \sum_{J \in \mathcal{D}}\left\langle f, \varphi_{I}\right\rangle\left\langle\varphi_{I}, S^{-1} \psi_{J}\right\rangle \psi_{J} \\
& =\sum_{J \in \mathcal{D}} \sum_{I \in \mathcal{D}}\left\langle f, \varphi_{I}\right\rangle\left\langle\varphi_{I}, S^{-1} \psi_{J}\right\rangle \psi_{J}=\sum_{J \in \mathcal{D}}\left\langle f, S^{-1} \psi_{J}\right\rangle \psi_{J} .
\end{aligned}
$$

To justify the shift of the order of the summation, it is sufficient to show that if $\eta \in \mathbb{S}_{\infty}$, then the series

$$
\sum_{J \in \mathcal{D}} \sum_{I \in \mathcal{D}}\left\langle f, \varphi_{I}\right\rangle\left\langle\varphi_{I}, S^{-1} \psi_{J}\right\rangle\left\langle\psi_{J}, \eta\right\rangle
$$

converges absolutely. To this end we note that since $\left(\left\langle f, \varphi_{I}\right\rangle\right)_{I \in \mathcal{D}} \in \dot{f}_{p q}^{s}$ and $\mathbf{B}$ is bounded on $\dot{f}_{p q}^{s}$, the sequence

$$
d:=\left(d_{J}\right)_{J \in \mathcal{D}} \quad \text { with } d_{J}:=\sum_{I \in \mathcal{D}}\left|\left\langle f, \varphi_{I}\right\rangle\right|\left|\left\langle\varphi_{I}, S^{-1} \psi_{J}\right\rangle\right|
$$

belongs to $\dot{f}_{p q}^{s}$. Using this fact one easily obtains (see $\underline{\underline{K}}$, Lemma 4.1 ) that,

$$
\sum_{J \in \mathcal{D}}\left|d_{J}\right|\left|\left\langle\psi_{J}, \eta\right\rangle\right|<\infty
$$

Theorem 3.3. Suppose that $s \in \mathbb{R}, 0<p, q \leq \infty$ and let $\Psi:=\left\{\psi_{I}\right\}_{I \in \mathcal{D}}$ be a frame for $L_{2}\left(\mathbb{R}^{d}\right)$ satisfying (2.6)-(2.8) for some $r>[s], k \geq \mathcal{N}$, and $M>\mathcal{J}$. Then $S^{-1} \Psi$ is a frame for $\dot{B}_{p q}^{s}$ or $\dot{F}_{p q}^{s}$, when $p<\infty$, if and only if the operator $\mathbf{B}$ defined by (3.3) is bounded on $\dot{b}_{p q}^{s}$ or $\dot{f}_{p q}^{s}$, respectively.

Proof. We again treat only the case of Triebel-Lizorkin spaces. Let us first assume that $\mathbf{B}$ is a bounded operator on $\dot{F}_{p q}^{s}$. We shall prove that there exist constants $A, B>0$ such that for $f \in \dot{F}_{p q}^{s}$,

$$
A\|f\|_{\dot{F}_{p q}^{s}} \leq\left\|\left(\left\langle f, S^{-1} \psi_{I}\right\rangle\right)_{I}\right\|_{\dot{f}_{p q}^{s}} \leq B\|f\|_{\dot{F}_{p q}^{s}} .
$$

The right-hand side estimate in (3.7) has already been established in (3.5).

To prove the left-hand-side estimate in (3.7) let us consider the operator $\mathbf{C}$ with matrix

$$
\mathbf{C}:=\left(c_{I J}\right)_{I, J \in \mathcal{D}}, \text { where } c_{I J}=\left\langle\psi_{J}, \varphi_{I}\right\rangle .
$$

As in (2.9) we know that $\mathbf{C}$ is bounded on $\dot{f}_{p q}^{s}$. Since

$$
\left\langle f, \varphi_{I}\right\rangle=\sum_{J \in \mathcal{D}}\left\langle f, S^{-1} \psi_{J}\right\rangle\left\langle\psi_{J}, \varphi_{I}\right\rangle
$$

for $I \in \mathcal{D}$, putting $c_{f}:=\left(\left\langle f, \varphi_{I}\right\rangle\right)_{I \in \mathcal{D}}$ and $d:=\left(\left\langle f, S^{-1} \psi_{I}\right\rangle\right)_{I \in \mathcal{D}}$, we can write

$$
\mathbf{C} d=c_{f} .
$$

Then

$$
\|f\|_{\dot{F}_{p q}^{s}} \leq c\left\|\left(\left\langle f, \varphi_{I}\right\rangle\right)_{I}\right\|_{\dot{f}_{p q}^{s}}=c\|\mathbf{C} d\|_{\dot{f}_{p q}^{s}} \leq c\|d\|_{\dot{f}_{p q}^{s}}=c\left\|\left(\left\langle f, S^{-1} \psi_{I}\right\rangle\right)_{I}\right\|_{\dot{f}_{p q}^{s}} .
$$


Assume now that $S^{-1} \Psi$ is a frame for $\dot{F}_{p q}^{s}$. We claim that the operator $\mathbf{B}$ (see (3.3) ) is necessarily bounded on $\dot{f}_{p q}^{s}$. To see this let $\left(d_{I}\right)_{I \in \mathcal{D}} \in \dot{f}_{p q}^{s}$. From (2.10) we know that the distribution $f=\sum_{I \in \mathcal{D}} d_{I} \varphi_{I}$ belongs to $\dot{F}_{p q}^{s}$ and

$$
\left\|\sum_{I \in \mathcal{D}} d_{I} \varphi_{I}\right\|_{\dot{F}_{p q}^{s}} \leq c\left\|\left(d_{I}\right)_{I}\right\|_{\dot{p}_{p q}^{s}}
$$

Since $S^{-1} \Psi$ is a frame for $\dot{F}_{p q}^{s}$, we also have

$$
\left\|\left(\left\langle f, S^{-1} \psi_{I}\right\rangle\right)_{I}\right\|_{\dot{f}_{p q}^{s}} \leq B\|f\|_{\dot{F}_{p q}^{s}} .
$$

Finally, we use $\left\langle f, S^{-1} \psi_{I}\right\rangle=\sum_{J \in \mathcal{D}} d_{J}\left\langle\varphi_{J}, S^{-1} \psi_{I}\right\rangle=\sum_{J \in \mathcal{D}} d_{J} b_{I J}=(\mathbf{B} d)_{I}$ and (3.8) -(3.9) to obtain

$$
\|\mathbf{B} d\|_{\dot{f}_{p q}^{s}}=\left\|\left(\left\langle f, S^{-1} \psi_{I}\right\rangle\right)_{I}\right\|_{\dot{f}_{p q}^{s}} \leq c\|f\|_{\dot{F}_{p q}^{s}} \leq c\|d\|_{\dot{f}_{p q}^{s}} .
$$

Although Theorem 3.3 is sufficient for our purposes, it does not answer the question, whether $\Psi$ is a frame itself for the Triebel-Lizorkin and Besov spaces. We address this question in the following proposition.

Proposition 3.4. Suppose $s \in \mathbb{R}, 0<p, q \leq \infty$ and let $\Psi:=\left\{\psi_{I}\right\}_{I \in \mathcal{D}}$ be a frame for $L_{2}\left(\mathbb{R}^{d}\right)$ satisfying (2.1) -(2.2) for some $r>\mathcal{N}, k \geq[s]$, and $M>\max \{\mathcal{J}, d+s\}$. If the operator $\mathbf{B}^{T}$ (the transpose of $\mathbf{B}$ ) is bounded on $\dot{b}_{p q}^{s}$ or $\dot{f}_{p q}^{s}$ for $p<\infty$, then $\Psi$ is a frame for $\dot{B}_{p q}^{s}$ or $\dot{f}_{p q}^{s}$ as well. In addition, if $f \in \dot{B}_{p q}^{s}\left(\right.$ or $f \in \dot{F}_{p q}^{s}$ with $\left.p<\infty\right)$, then

$$
f=\sum_{I \in \mathcal{D}}\left\langle f, \psi_{I}\right\rangle S^{-1} \psi_{I}
$$

in the sense of $\mathbb{S}^{\prime} / \mathcal{P}$.

The proof of this proposition is quite similar to the proofs of Lemma 3.2 and Theorem 3.3 and will be omitted.

We next prove that if $\Psi$ satisfies $\mathbf{A} \mathbf{1}$ and $\mathbf{A} \mathbf{2}$ for sufficiently small $\varepsilon>0$, then $\mathbf{B}$ is indeed a bounded operator on $\dot{b}_{p q}^{s}$ and $\dot{f}_{p q}^{s}$.

Theorem 3.5. Suppose $s \in \mathbb{R}, 0<p, q \leq \infty$, and let $\Psi:=\left\{\psi_{I}\right\}_{I \in \mathcal{D}}$ be a family of functions satisfying $\mathbf{A} 1$ and $\mathbf{A 2}$ for some $r, k>\max \{[s], \mathcal{N}\}$ and $M>$ $\max \{\mathcal{J}, d+s\}$. If $\varepsilon>0$ is sufficiently small, then $S^{-1} \Psi$ is a frame for $\dot{B}_{p q}^{s}$ and $\dot{F}_{p q}^{s}$, if $p<\infty$.

Proof. According to Theorem 3.3 we need to establish that for sufficiently small $\varepsilon>0$ the operator B defined in (3.3) is bounded on $\dot{f}_{p q}^{s}$ or $\dot{b}_{p q}^{s}$, respectively.

We begin by showing that the operator $S^{-1}$ is bounded on $\dot{F}_{p q}^{s}$. To this end it suffices to show that

$$
\|I-S\|_{\dot{F}_{p q}^{s} \rightarrow \dot{F}_{p q}^{s}}<1 .
$$

In particular, we shall show that there exists a constant $c>0$ independent of $\varepsilon$ such that for $f \in \dot{F}_{p q}^{s}$,

$$
\|(I-S) f\|_{\dot{F}_{p q}^{s}} \leq c \varepsilon\|f\|_{\dot{F}_{p q}^{s}} .
$$

According to (2.12) it suffices to prove that the operator $\mathbf{D}$ with matrix

$$
\mathbf{D}:=\left(d_{I J}\right)_{I, J \in \mathcal{D}}, \text { where } d_{I J}:=\left\langle(I-S) \varphi_{J}, \varphi_{I}\right\rangle,
$$


is bounded on $\dot{f}_{p q}^{s}$ and

$$
\|\mathbf{D} d\|_{\dot{f}_{p q}^{s}} \leq c \varepsilon\|d\|_{\dot{f}_{p q}^{s}} \quad \text { for } d \in \dot{f}_{p q}^{s} .
$$

Using that $\varphi_{J}=\sum_{Q}\left\langle\varphi_{J}, \varphi_{Q}\right\rangle \varphi_{Q}$ and $S \varphi_{J}=\sum_{Q}\left\langle\varphi_{J}, \psi_{Q}\right\rangle \psi_{Q}$, we have

$$
\begin{aligned}
d_{I J} & =\left\langle(I-S) \varphi_{J}, \varphi_{I}\right\rangle=\sum_{Q}\left\langle\varphi_{J}, \varphi_{Q}\right\rangle\left\langle\varphi_{Q}, \varphi_{I}\right\rangle-\sum_{Q}\left\langle\varphi_{J}, \psi_{Q}\right\rangle\left\langle\psi_{Q}, \varphi_{I}\right\rangle \\
& =\sum_{Q}\left\langle\varphi_{J}, \varphi_{Q}\right\rangle\left\langle\varphi_{Q}-\psi_{Q}, \varphi_{I}\right\rangle+\sum_{Q}\left\langle\varphi_{J}, \varphi_{Q}-\psi_{Q}\right\rangle\left\langle\psi_{Q}, \varphi_{I}\right\rangle .
\end{aligned}
$$

Setting now

we obtain

$$
\begin{aligned}
& \mathbf{D}_{1}:=\left(d_{I J}^{1}\right)_{I, J \in \mathcal{D}}, \text { where } d_{I J}^{1}:=\left\langle\varphi_{J}-\psi_{J}, \varphi_{I}\right\rangle, \\
& \mathbf{D}_{2}:=\left(d_{I J}^{2}\right)_{I, J \in \mathcal{D}}, \text { where } d_{I J}^{2}:=\left\langle\varphi_{J}, \varphi_{I}\right\rangle, \\
& \mathbf{D}_{3}:=\left(d_{I J}^{3}\right)_{I, J \in \mathcal{D}}, \text { where } d_{I J}^{3}:=\left\langle\psi_{J}, \varphi_{I}\right\rangle, \\
& \mathbf{D}_{4}:=\left(d_{I J}^{2}\right)_{I, J \in \mathcal{D}}, \text { where } d_{I J}^{4}:=\left\langle\varphi_{J}, \varphi_{I}-\psi_{I}\right\rangle,
\end{aligned}
$$

$$
\mathbf{D}=\mathbf{D}_{1} \mathbf{D}_{2}+\mathbf{D}_{3} \mathbf{D}_{4}
$$

The operators associated with all four matrices are bounded on $\dot{f}_{p q}^{s}$. Indeed, note that $\mathbf{D}_{1}, \mathbf{D}_{2}$, and $\mathbf{D}_{3}$ give rise to bounded operators as for the operator $\mathbf{C}$ in (2.9) because the families $\Phi, \Psi$, and $\left\{\varepsilon^{-1}\left(\varphi_{I}-\psi_{I}\right)\right\}_{I \in \mathcal{D}}$ consist of smooth molecules. In particular, the last family gives us that for $d \in \dot{f}_{p q}^{s}$,

$$
\left\|\mathbf{D}_{1} d\right\|_{\dot{f}_{p q}^{s}} \leq c \varepsilon\|d\|_{\dot{f}_{p q}^{s}}
$$

As far as $\mathbf{D}_{4}$ is concerned, since $\left\{\varepsilon^{-1}\left(\varphi_{I}-\psi_{I}\right)\right\}_{I \in \mathcal{D}}$ is a norming family, then as for the operator $\mathbf{A}$ defined in (2.3) we obtain that for $d \in \dot{f}_{p q}^{s}$,

$$
\left\|\mathbf{D}_{4} d\right\|_{\dot{f}_{p q}^{s}} \leq c \varepsilon\|d\|_{\dot{f}_{p q}^{s}} .
$$

Taking into account (3.13)-(3.14) it follows that for $d \in \dot{f}_{p q}^{s}$,

$$
\|\mathbf{D} d\|_{f_{p q}^{s}} \leq c\left(\left\|\mathbf{D}_{1}\right\|\left\|\mathbf{D}_{2}\right\|+\left\|\mathbf{D}_{3}\right\|\left\|\mathbf{D}_{4}\right\|\right)\|d\|_{\dot{f}_{p q}^{s}} \leq c \varepsilon\|d\|_{\dot{f}_{p q}^{s}}
$$

which establishes (3.12). Consequently, (3.11) holds and hence for sufficiently small $\varepsilon>0$ the operator $S^{-1}$ is bounded on $\dot{F}_{p q}^{s}$, i.e. for $f \in \dot{F}_{p q}^{s}$,

$$
\left\|S^{-1} f\right\|_{\dot{F}_{p q}^{s}} \leq c\|f\|_{\dot{F}_{p q}^{s}} .
$$

Finally, in order to show that the operator $\mathbf{B}$ is bounded on $\dot{f}_{p q}^{s}$ we recall from (2.10) that if $d:=\left(d_{I}\right)_{I \in \mathcal{D}} \in \dot{f}_{p q}^{s}$ and $f=\sum_{I} d_{I} \varphi_{I}$, then

$$
\|f\|_{\dot{F}_{p q}^{s}} \leq c\|d\|_{\dot{f}_{p q}^{s}} .
$$

Clearly, for $I \in \mathcal{D}$,

$$
\left\langle S^{-1} f, \psi_{I}\right\rangle=\sum_{J \in \mathcal{D}} d_{J}\left\langle S^{-1} \varphi_{J}, \psi_{I}\right\rangle=\sum_{J \in \mathcal{D}} d_{J}\left\langle\varphi_{J}, S^{-1} \psi_{I}\right\rangle=(\mathbf{B} d)_{I} .
$$

Therefore,

$$
\|\mathbf{B} d\|_{\dot{f}_{p q}^{s}}=\left\|\left(\left\langle S^{-1} f, \psi_{I}\right\rangle\right)_{I}\right\|_{\dot{f}_{p q}^{s}} \leq c\left\|S^{-1} f\right\|_{\dot{F}_{p q}^{s}} \leq c\|f\|_{\dot{F}_{p q}^{s}} \leq c\|d\|_{\dot{f}_{p q}^{s}} .
$$

Here, for the first inequality, we used that $\Psi$ is a frame for $\dot{F}_{p q}^{s}$ if $\varepsilon>0$ is sufficiently small, which follows by Theorem 3.1) we also used (3.15)-(3.16). 


\section{REFERENCES}

[BL] J. Benedetto, S. Li, The theory of multiresolution analysis frames and applications to filter banks, Appl. Comp. Harm. Anal., 5 (1998), 389-427. MR.1646534 (99k:42054)

[De] R. DeVore, Nonlinear approximation, Acta Numer. (1998), 51-150. MR.1689432 (2001a:41034)

[Do] D. Donoho, Unconditional bases are optimal bases for data compression and for statistical estimation, Appl. Comp. Harm. Anal., 1 (1993), 100-115. MR.1256530 (94j:94011)

[DS] R. J. Duffin, A. C. Schaeffer, A class of nonharmonic Fourier series, Trans. Amer. Math. Soc., 72 (1952), 341-366. MR0047179 (13:839a)

[FG1] H. G. Feichtinger, K. Gröchenig, A unified approach to atomic decompositions via integrable group representations, Lect. Notes Math. 132, Berlin-Heidelberg-New York: Springer 1988, pp. 52-73. MR0942257 (89h:46035)

[FG2] H. G. Feichtinger, K. Gröchenig, Gabor frames and time frequency analysis of distributions, J. Functional Anal., 146 (1997), 464-495. MR.1452000 (98k:42041)

[FJ1] M. Frazier, B. Jawerth, Decomposition of Besov Spaces, Indiana Univ. Math. J., 34 (1985), 777-799. MR0808825 (87h:46083)

[FJ2] M. Frazier, B. Jawerth, A discrete transform and decompositions of distribution, J. of Functional Analysis, 93 (1990), 34-170. MR1070037 (92a:46042)

[FJW] M. Frazier, B. Jawerth, and G. Weiss, Littlewood-Paley Theory and the Study of Function Spaces, CBMS 79 (1991), Amer. Math. Soc., Providence, RI. MR.1107300 (92m:42021)

[G] K. Gröchenig, Discribing Functions: Atomic Decompositions Versus Frames, Monatsh. Math., 112 (1991), 1-41. MR1122103 (92m:42035)

[Gr] L. Grafakos, Classical and Modern Fourier Analysis, Prentice Hall 2004.

[HW] C. E. Heil, D. F. Walnut, Continuous and Discrete wavelet transforms, SIAM Review, 31 (1989), 628-666. MR 1025485 (91c:42032)

[K] G. Kyriazis, Decomposition systems for Function spaces, Studia Math., 157 (2003), 133169. MR1981430 (2004e:42036)

[KP] G. Kyriazis, P. Petrushev, New Bases for Treibel-Lizorkin and Besov spaces, Trans. Amer. Math. Soc., 354 (2002), 749-776. MR1862566 (2002k:46082)

[Pee] J. Peetre, New thought on Besov spaces, Duke Univ. Math. Series, Durham, N.C., 1993. MR0461123 (57:1108)

[Pet] P. Petrushev, Bases consisting of rational functions of uniformly bounded degrees or more general functions, J. Funct. Anal., 174 (2000), 18-75. MR1761363 (2001k:46016)

[T] H. Triebel, Theory of Function Spaces, Birkhauser, 1993. MR0781540 (86j:46026)

Department of Mathematics and Statistics, University of Cyprus, 1678 Nicosia, CYPRUS

E-mail address: kyriazis@ucy.ac.cy

Department of Mathematics, University of South Carolina, Columbia, South CarOLINA 29208

E-mail address: pencho@math.sc.edu 